\title{
Evaluación de la eficiencia de tres equipos de extracción de aceite con diferentes genotipos de palma aceitera (Elaeis sp.)
}

\section{(Evaluation of the efficiency of three oil extraction equipments with different oil palm genotypes (Elaeis sp.))}

\author{
Mercedes Navarrete Parraga, ${ }^{1}$ Silvia Zambrano Marcillo, ${ }^{1}$ Walter Zambrano Sabando, ${ }^{1}$ Martha Romero \\ Pizarro, ${ }^{1}$ Marcelo Racines Jaramillo, ${ }^{1}$ Ernesto Paredes Puga, ${ }^{2}$ Leonardo Quintero Román, ${ }^{3}$ Digner \\ Ortega Cedillo ${ }^{1}$
}

\section{Resumen}

Este trabajo se realizó en el Laboratorio de extracción de aceite del Programa de Palma Africana de la Estación Experimental "Santo Domingo" del INIAP, el objetivo fue determinar la eficiencia de tres equipos de extracción de aceite, en tres genotipos de palma aceitera. Los equipos utilizados fueron: extractor de solvente Velp, extractor Soxhlet y la prensa hidráulica de 1500 psi. Los genotipos evaluados forman parte del Banco de Germoplasma del Programa de Palma: palma africana (Elaeis guineensis), palma americana (Elaeis oleífera) y el híbrido interespecífico (E. oleífera XE. guineensis). El diseño experimental utilizado fue un diseño completo al azar (DCA), con 20 observaciones, asistido por las pruebas de significancia de t de student y Tukey al $5 \%$, para determinar diferencias entre medias de tratamientos. Los resultados mostraron diferencias altamente significativas entre los diferentes factores evaluados. El mayor porcentaje de aceite por racimo se obtuvo con el genotipo E. guineensis en el extractor Soxhlet $(18,43 \%)$ y el menor porcentaje para E. oleífera con la prensa hidráulica (0,34\%). Realizado el análisis de costos de los tratamientos, se determinó que el menor costo se obtuvo con la prensa hidráulica con 8.20 dólares por muestra de extracción de aceite.

\section{Palabras clave}

Grasa, material, mesocarpio, métodos, solvente

\begin{abstract}
This work was performed in the oil extraction laboratory of the African Palm Program, of the Experimental Station "Santo Domingo" of the INIAP. The main objective was to determine the efficiency of three oil extraction equipments, in three oil palm genotypes. The equipments used were Velp solvent extractor, Soxhlet extractor and a 1500 psi hydraulic press. The genotypes evaluated are part of the germplasm bank of the Palm Program at INIAP: Elaeis guineensis (african palm), Elaeis oleifera (american palm) and E. oleifera x E. guineensis (interspecific hybrid). The experimental design was a complete random design (DCA), with 20 observations, followed by student's test and Tukey test at a 5 significance, to determine differences between treatment means. The results showed highly significant differences between the different factors evaluated. The higher percentage of oil per bunch was obtained with the genotype E. guineensis in the Soxhlet extractor (18.43\%) and the lowest percentage for E. oleifera with the hydraulic press $(0.34 \%)$. The analysis of costs determined that the lowest cost was obtained with the hydraulic press with USD 8,20 per sample.
\end{abstract}

\section{Keywords}

Grease, Material, Mesocarp, Methods, Solvent.

1 Instituto Nacional de Investigaciones Agropecuarias, La Concordia-Ecuador (mercedes.navarrete, silvia.zambrano, walter. zambrano, alicia.romero, marcelo.racines, digner.ortega@iniap.gob.ec)

2 Ernesto Paredes, San Juan-Ecuador (paredespuga12@gmail.com)

3 Leonardo Quintero, Santo Domingo-Ecuador (l.quintero.roman@gmail.com) 


\section{Introducción}

La palma aceitera (Elaeis guineensis Jacq.) es una planta tropical, propia de climas cálidos, en Ecuador tiene una trayectoria productiva por más de 60 años. El cultivo actualmente representa el $5 \%$ de la superficie total destinada a la producción agropecuaria del país, siendo el segundo cultivo en extensión después del arroz. Existen 257120.93 ha sembradas en 8149 plantaciones, siendo la provincia de Esmeraldas la de mayor superficie sembrada con 116430.48 ha, seguida por Los Ríos con 39146.08 ha, otras 101387.92 ha y Santa Elena la menor superficie con 156.45 ha (Asociación Nacional de Cultivadores de Palma Aceitera, 2017).

Los frutos son carnosos y forman un racimo, en estado maduro son cosechados y llevados a las plantas extractoras de aceite, donde después de varios procesos físicos y químicos, se extrae el aceite rojo, que es utilizado en la industria alimenticia humana y animal, también es utilizado como biocombustible y algunos de los subproductos y/o residuos resultantes en el proceso de extracción sirven como abono para las plantas (Calvache, 2013).

El Programa de Palma Africana de la Estación Experimental Santo Domingo, Instituto Nacional de Investigaciones Agropecuarias (INIAP) desde su creación de manera continua desarrolla investigaciones para obtener nuevos materiales de palma de aceite, con características deseables, tales como: alto rendimiento de fruta fresca y de aceite, tolerancia o resistencia a plagas (insectos y enfermedades), lento crecimiento, entre otras.

Por lo tanto, esta investigación tuvo como objetivo evaluar a nivel de laboratorio la eficiencia de tres equipos de extracción de aceite con diferentes genotipos de palma aceitera, en el cantón La Concordia, provincia de Santo Domingo de Los Tsáchilas.

\section{Metodología}

El presente trabajo se realizó en el Laboratorio de Extracción de Aceite del Programa de Palma Africana de la Estación Experimental Santo Domingo del INIAP, ubicada en el km 38 de la vía Santo Domingo,-Quinindé, localizado geográficamente en las coordenadas $79^{\circ} 22^{\prime}$ de longitud oeste y $00^{\circ} 01^{\prime}$ de latitud norte, a $300 \mathrm{msnm}$.

Se utilizaron genotipos de edades comprendidas entre 8 a 20 años, del Banco de Germoplasma del Programa de Palma Africana de la EESD, como: Elaeis guineensis, Elaeis oleiffera y Elaeis oleífera x Elaeis guineensis, Los equipos de extracción utilizados en este estudio fueron: Extractor de solvente Velp, Extractor de Soxhlet y Prensa hidráulica a 1500 psi.

Los tratamientos obtenidos de un factorial $3 \times 3$, fueron analizados a través de un diseño completo al azar, con la asistencia de la prueba de Tukey al $5 \%$ para determinar diferencia entre medias de tratamientos. Además, se utilizó la prueba de t student para determinar diferencia entre las medias de los equipos de extracción de aceite y de los genotipos de palma evaluados.

Se realizó un análisis de costos de extracción utilizando el método ABC (costeo basado en actividades), aplicado a ensayos de laboratorio. 


\section{Manejo del experimento}

\section{Análisis físico de los racimos (Prada y Romero, 2012).}

Previo a la obtención del porcentaje de aceite en cada uno de los materiales se realizó el análisis físico de racimos que consistió en los siguientes pasos: se pesó el racimo que viene del campo, se despicó y se contaron todas las espigas, se pesó el raquis y pesaron dos muestras, una de 2 $\mathrm{kg}$ y otra de $5 \mathrm{~kg}$.

\section{Muestra de 2 kilogramos, procedimiento}

a. De esta muestra se pesó 250 gramos de frutos fértiles y se contó el número de frutos.

b. Se procedió a despulpar los frutos, obteniendo mesocarpio y nuez fresca.

c. Se registró el peso del mesocarpio y la nuez fresca de cada muestra.

d. El mesocarpio paso a una estufa por 24 horas y la nuez por cuatro horas, las muestras a una temperatura de $105^{\circ} \mathrm{C}$.

e. Transcurrida las 4 horas en la estufa, se pesó la nuez para obtener el peso seco.

f. Se procedió a partir la nuez para obtener la almendra y el cuesco.

g. Se pesaron y se contaron las almendras, ya que muchas semillas pueden tener doble almendra o carecer de esta.

h. Con un calibrador (pie de rey) se midió el grosor del cuesco.

i. Después que el mesocarpio pasó las 24 horas en la estufa, se procedió a pesar y así obtener el peso seco del mesocarpio. Este mesocarpio seco se molió para realizar el análisis en el extractor Velp y Soxhlet.

\section{Muestra de 5 kilogramos, procedimiento:}

a. Esta muestra se dejó por cinco días para desprender los frutos de las espigas.

b. Transcurrido los cinco días se procedió a clasificar los frutos como: fértiles, partenocárpicos, abortados, blancos.

c. Se contaron y se pesaron los frutos fértiles, partenocárpicos, blancos y abortados.

d. Los frutos fértiles fueron utilizados para realizar el análisis en la prensa hidráulica.

\section{Procedimiento para la obtención del porcentaje de extracción de aceite}

\section{Equipo de extractor de solvente Velp (Metodología desarrollada en el Programa de Palma Africana, EESD).}

En una balanza se pesó 5 gramos de mesocarpio seco y molido, se colocó en papel filtro y todo en un dedal, esta muestra va dentro del vaso con el disolvente (Éter de petróleo). Este disolvente comenzó a calentar y a hervir a $120^{\circ} \mathrm{C}$, hasta que se complete la extracción del aceite de la muestra; siendo 90 minutos de inmersión, 10 minutos de lavado y 40 minutos de recuperación del disolvente a $170^{\circ} \mathrm{C}$. 
Equipo extractor Soxhlet (Prada y Romero, 2012).

En una balanza se pesó 5 gramos de mesocarpio seco y molido, y se colocó en un dedal de papel filtro que se sitúa en la cámara del extractor soxhlet (camisa). Se calentó el disolvente éter de petróleo en una manta calentadora, contenido en el balón de $500 \mathrm{ml}$.

El disolvente químico, durante la extracción de aceite sigue un circuito cerrado pasando del balón a la cámara de condensación en forma de vapor y de la cámara de condensación pasa al recipiente de extracción condensada en forma de gotas. Del recipiente de extracción, el disolvente regresa al balón por el sistema de vasos comunicantes. Este proceso se repite hasta que se complete la extracción de aceite de la muestra; es decir hasta que el disolvente presente su coloración inicial (transparente).

En estos dos equipos, después de realizada la extracción se procedió a apagar la plancha y se la dejó enfriar. Se desmontó el sistema, el solvente se recuperó y se almacenó para ser utilizado nuevamente, los dedales humedecidos con solvente, se colocaron en una estufa a $105^{\circ} \mathrm{C}$, para retirar la totalidad del solvente, luego fueron retirados de la estufa y se dejaron enfriar en un desecador para posteriormente pesar, el aceite se cuantificó por diferencia.

Para obtener el porcentaje de extracción de aceite en los dos procedimientos antes descritos se utilizó la siguiente fórmula:

$$
\% A C / R=\frac{\% \text { de fruto por racimo } x \% \text { pulpa por fruto } x \% \text { aceite pulpa fresca }}{10.000}
$$

\section{Prensa hidráulica (Metodología desarrollada en el Programa de Palma Africana, EESD).}

En una balanza se pesó $1 \mathrm{~kg}$ de fruto fértil de palma aceitera, los mismos que fueron autoclavados por 55 minutos $a 110^{\circ} \mathrm{C}$, con el objeto que los frutos se cocinen. Posteriormente los frutos se colocaron en la prensa hidráulica, se hace presión y se obtuvo el aceite, finalmente se realizó el decantado de la muestra obtenida de la prensa, esta muestra se pesó y se obtuvo el porcentaje de aceite por racimo.

El porcentaje de extracción de aceite por racimo se determinó mediante la siguiente ecuación:

$$
n=\frac{A c}{M a} \quad x \quad 100
$$

Dónde:

$\mathrm{N}=$ Rendimiento (\%)

Ac = Cantidad de aceite crudo extraído $(\mathrm{g})$

$\mathrm{Ma}=$ Cantidad de materia prima utilizada $(\mathrm{g})$

\section{Análisis de costos de procesamiento de los equipos en estudio}

Se realizó un análisis comparativo de costos mediante el método de costeo basado en actividades (ABC) (Adame, 2000). Se consideró el tiempo de procesamiento de extracción por cada equipo, el 
costo del uso de los equipos (precio del equipo, depreciación, mantenimiento); los costos directos (materia prima, mano de obra, etc.), y los costos indirectos (vestuario, energía, material de aseo).

\section{Resultados y discusión}

Los resultados observados en tabla 1, muestran los valores del análisis de varianza del porcentaje de aceite por racimo extraído. El cuadrado medio de equipos fue de 54.00 y la F calculada de 175,66. El cuadrado medio para genotipo fue de 32.40 y una F calculada de 105.40. Existiendo diferencias altamente significativas entre tratamientos. Con un coeficiente de variación de 18.81.

Tabla 1. Análisis de Varianza* porcentaje de aceite por racimo. EESD, 2018

\begin{tabular}{|l|l|l|l|l|l|}
\hline \multicolumn{1}{|c|}{ Fuente de Variación } & \multicolumn{1}{c|}{ GI } & \multicolumn{1}{c|}{ SC } & \multicolumn{1}{c|}{ CM } & \multicolumn{1}{c|}{ FC } & \multicolumn{1}{c|}{ p-Valor } \\
\hline Equipos & 2 & 108.00 & 54.00 & 175.66 & $* *$ \\
\hline Genotipos & 2 & 64.81 & 32.40 & 105.40 & $* *$ \\
\hline Error & 175 & 53.80 & 0.31 & & \\
\hline Total & 179 & 226.61 & & & \\
\hline CV. 18.81 & $R^{2} .0 .76$ & & \\
\hline
\end{tabular}

*Datos originales transformado a $\sqrt{ } \mathrm{x}+1$

En la tabla 2 se observan las medias, comparadas a través de la prueba de t entre equipos y genotipos utilizados, donde el equipo 2 (Soxhlet) con el material E. guineensis fue el de mayor porcentaje de aceite extraído por racimo (18.43\%), este mismo genotipo guineensis es el que obtuvo mayor porcentaje de aceite en cada uno de los equipos en estudio. El genotipo oleífera fue el de menor porcentaje de extracción, con todos los equipos evaluados, estos resultados concuerda con Alvarado (2010), donde manifiesta que esta especie es inferior a E. guineensis en relación a su capacidad de producción de aceite.

El resultado de extracción de aceite obtenido del genotipo E. oleífera x E. guineensis, utilizando el extractor soxhlet (equipo) fue de $13.84 \%$ de aceite por racimo (tabla 2), lo que concuerda con Alvarado (2010), que menciona que este material tiene un contenido de aceite en el racimo mayor al $12 \%$.

Tabla 2. Medias entre equipos de extracción de aceite y genotipos de palma. EESD, 2018

\begin{tabular}{|c|c|c|c|c|}
\hline Equipos & Genotipo* & Variable & $\mathrm{n}$ & Media \\
\hline \multirow{3}{*}{$\begin{array}{l}1 \text { (Extractor de solven- } \\
\text { te Velp) }\end{array}$} & 1 & \% de aceite por racimo & 20 & 15.58 \\
\hline & 2 & $\%$ de aceite por racimo & 20 & 6.03 \\
\hline & 3 & $\%$ de aceite por racimo & 20 & 10.64 \\
\hline \multirow{3}{*}{ 2. (Soxhlet) } & 1 & $\%$ de aceite por racimo & 20 & 18.43 \\
\hline & 2 & $\%$ de aceite por racimo & 20 & 7.00 \\
\hline & 3 & $\%$ de aceite por racimo & 20 & 13.84 \\
\hline \multirow{3}{*}{ 3. (Prensa hidráulica) } & 1 & $\%$ de aceite por racimo & 20 & 6.18 \\
\hline & 2 & $\%$ de aceite por racimo & 20 & 0.34 \\
\hline & 3 & $\%$ de aceite por racimo & 20 & 2.49 \\
\hline
\end{tabular}


Al comparar los equipos de extracción de aceite se encontró que el equipo Soxhlet obtuvo la mayor cantidad de aceite extraído con $13.09 \%$ de aceite por racimos, como se observa en la figura 1, resultados que concuerdan con Arancibia y Calero (2011) quienes obtuvieron mayor eficiencia en la extracción de aceite con el uso de solvente.

La prensa hidráulica fue la que extrajo menor cantidad de aceite por racimo (3.00 \%), estos resultados difieren de los obtenidos por Nolasco (2015), donde la extracción mecánica tuvo una mayor producción de aceite, al evaluar cuatro métodos de extracción de aceite en semilla de piñón.

Al analizar los resultados a través del análisis de varianza se encontraron tres rangos de significación, por lo tanto, existieron diferencias significativas entre los equipos de extracción de aceite (figura 1).

Figura 1. Medias de extracción de aceite según los equipos estudiados. EESD, 2018

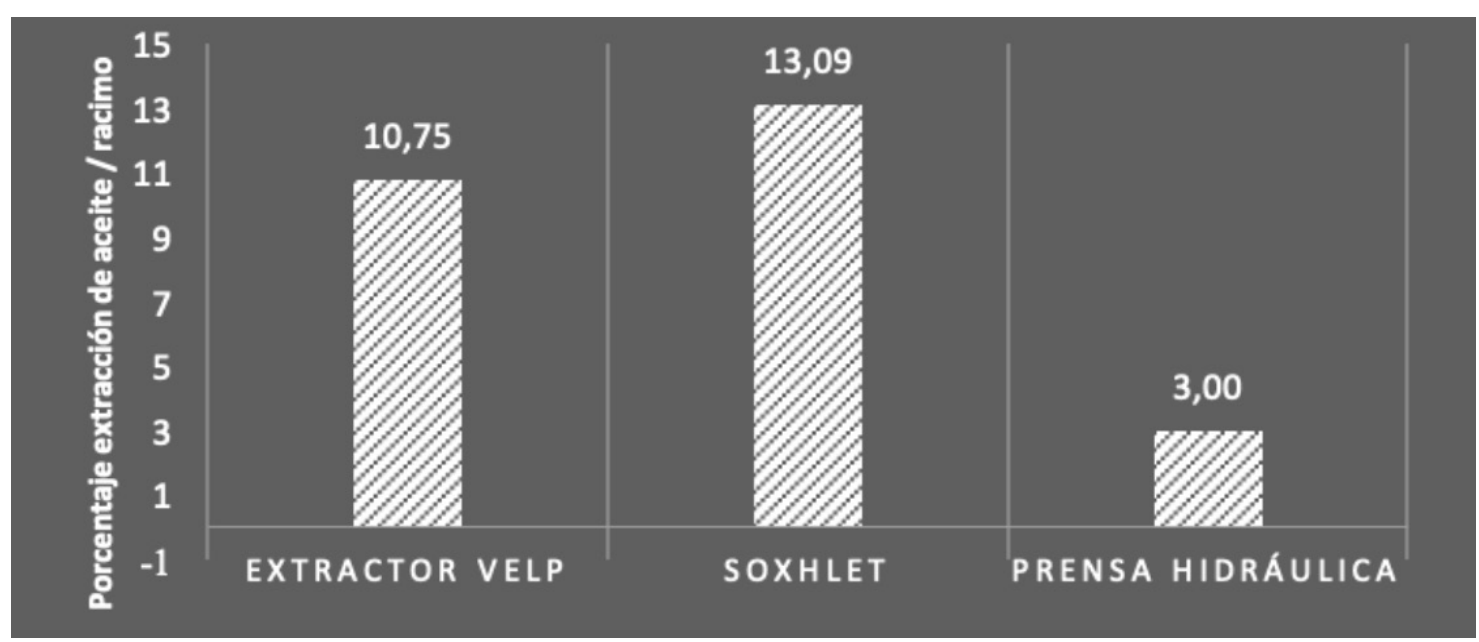

En la figura 2, se observan tres rangos de significación, existiendo diferencias en la extracción de aceite entre los tres genotipos de palma aceitera evaluados. El genotipo E. guineensis fue el que obtuvo la mayor cantidad de aceite extraído con $13.40 \%$ de aceite/racimo, utilizando Guineensis Dura y Tenera, lo que concuerda con Trávez (2015), que en su estudio comparó el hibrido OXG con E. guineensis, obteniendo el más alto porcentaje de extracción en E. guineensis (Tenera) con $22.28 \% /$ racimo.

El porcentaje de aceite por racimo en E. oleiffera fue de $4.46 \%$ y OxG fue $8.99 \%$, lo que concuerda con Vieira et al. (2012), que en sus estudios realizados obtuvieron resultados en $E$. oleífera de $1.7 \%$ a $4.4 \%$ y en el híbrido interespecífico de $3.4 \%$ a $17 \%$.

En estudios realizados en palma E. oleífera nativa de la Amazonía ecuatoriana por Paredes (2015), manifiesta que el porcentaje de extracción de aceite por racimo de esta especie fue de $3.90 \%$, lo que concuerda con los resultados de esta investigación.

Los costos por muestras de cada uno de los equipos de extracción se muestran en la tabla 3 , donde el menor costo se obtuvo con la prensa hidráulica con 8.20 dólares, y el mayor costo con la utilización del extractor Velp con 12.86 dólares. Resultados que concuerda con Rodríguez, Alcaraz y Real (2012) y (Mercer y Armenta 2011), quienes manifiestan que los extractores de solventes se utilizan a escala de laboratorio, a nivel industrial resultan costoso por el valor comercial de los solventes, además se obtienen esencias impurificadas con otras sustancias. En la actualidad se requiere que los procesos de extracción sean limpios y amigables con el ambiente. 
Figura 2. Medias de extracción de aceite según los genotipos de palma aceitera estudiados. EESD, 2018

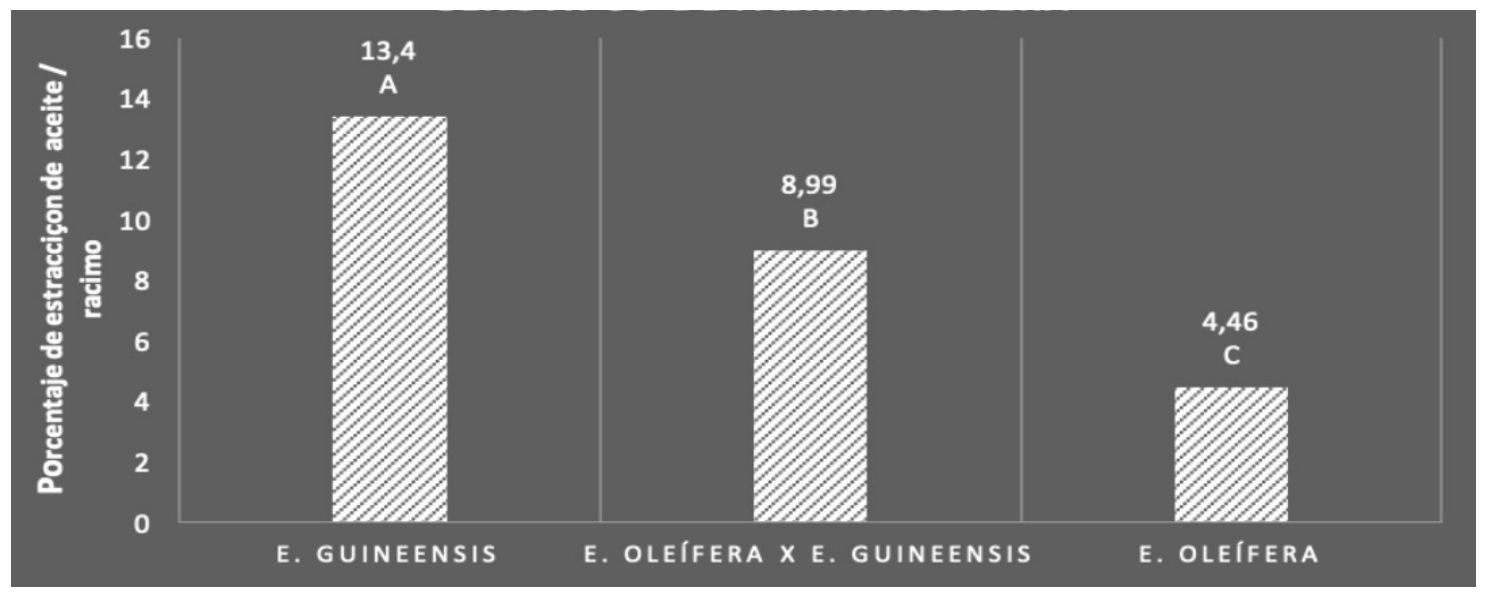

Al respecto, Méndez (2012) menciona que las prensas hidráulicas son mejores por el costo mínimo, su flexibilidad y no pasa mucho tiempo en los ajustes del equipo automatizado, además, son bastantes sencillas en su diseño, con pocas partes en movimiento y están siempre lubricadas con un fluido de aceite bajo presión.

Tabla 3. Costos / muestra (USD) de extracción en cada uno de los equipos estudiados. EESD, 2018.

\begin{tabular}{|l|l|l|l|}
\hline \multicolumn{1}{|c|}{ Costos y Gastos } & \multicolumn{1}{|c|}{ Extractor Velp } & \multicolumn{1}{|c|}{$\begin{array}{c}\text { Extractor } \\
\text { Soxhlet }\end{array}$} & \multicolumn{1}{|c|}{$\begin{array}{c}\text { Prensa } \\
\text { hidráulica }\end{array}$} \\
\hline $\begin{array}{l}\text { Subtotal Infraestructura, equipos, materiales } \\
\text { larga duración }\end{array}$ & 1.69 & 1.56 & 1.30 \\
\hline Subtotal costos directos & 10.65 & 9.92 & 6.69 \\
\hline Subtotal costos indirectos & 0.52 & 0.54 & 0.21 \\
\hline Costos / Muestras (USD) & 12.86 & 12.02 & 8.20 \\
\hline
\end{tabular}

\section{Conclusiones y recomendaciones}

\subsection{Conclusiones}

Los métodos de extracción de aceite utilizando solventes químicos extraen mayor porcentaje de aceite de fruta fresca de palma, sin embargo estos son útiles únicamente a nivel de laboratorio y no a nivel agroindustrial.

\subsection{Recomendaciones}

- A nivel de laboratorio se recomienda el uso del equipo Soxhlet, por extraer la mayor cantidad de aceite en los tres genotipos utilizados.

- Establecer el punto de madurez óptimo en los genotipos Oleífera x Guineensis y Oleíferas, con la finalidad de obtener la mayor concentración de aceite/racimo. 
- Realizar nuevos estudios clasificando los genotipos Guineensis y Oleíferas de acuerdo a su origen.

\section{Agradecimiento}

Al Instituto Nacional de Investigaciones Agropecuarias (INIAP), por el apoyo brindado en la realización de la presente investigación. Este artículo forma parte del II Simposio Internacional Producción Integrada de Frutas 2019.

\section{Bibliografía}

Adame, R. (2000). Costeo basado en actividades (ABC), conceptos, teoría y metodología de implementación. (Tesis de Maestría). Universidad Autónoma de Nuevo León. Monterrey, Nuevo León, México.

Alvarado, A. (2010). Avances en el mejoramiento genético de la palma de aceite en Centroamérica. Revista Palmas, 31(especial): 126-143.

Arancibia, Y. y Calero, T. (2011). Obtención de Biodiesel a Partir del Aceite de Semillas Oleaginosas de la Provincia de Chimborazo (Bachelor's thesis). (Tesis de grado). Escuela Superior Politécnica de Chimborazo, Riobamba.

Asociación Nacional de Cultivadores de Palma Aceitera (ANCUPA). (2017). Censo Nacional Palmero 2017. Memoria Técnica. Inventario de Plantaciones de Palma Aceitera en el Ecuador. Quito, Ecuador. $54 \mathrm{p}$.

Calvache, M. (2013). La competitividad del aceite de palma africana ecuatoriano en el mercado internacional, período 2006-2010. (Tesis de grado). Pontificia Universidad Católica del Ecuador. Quito, Ecuador.

Méndez, L. (2012). Diseño e implementación de una prensa hidráulica para la extracción de aceites fijos, en el laboratorio de investigaciones de extractos vegetales (LIEXVE) del centro de investigaciones de la Facultad de Ingeniería de la Universidad de San Carlos de Guatemala. (Tesis de grado). Universidad de San Carlos de Guatemala.

Mercer, P.,y Armenta, R. (2011). Developments in oil extraction from microalgae. European journal of lipid science and technology, 113 (5): 539-547.

Nolasco, E. (2015). Evaluación de cuatro métodos de extracción de aceite de la semilla de piñón (Jatropha curcas) var. Cabo Verde y cuantificación de sus ésteres de forbol. (Tesis de Grado). Escuela Agrícola Panamericana, Zamorano, Honduras.

Paredes, E. (2015). Estudio de la variedad genética de cinco accesiones de palma Americana (Elaeis oleífera HBK) nativa de la amazonia ecuatoriana. (Tesis de grado). Universidad de Guayaquil. Guayaquil, Ecuador.

Prada, F. y Romero, H. (2012). Muestreo y análisis de racimos en el cultivo de la palma de aceite. Tecnologías para la agroindustria de la palma de aceite: guía para facilitadores. Centro Nacional de Investigación en Palma de Aceite (Cenipalma) Bogotá, DC:. Colombia.

Rodríguez, M., Alcaraz L. y Real, S. (2012). Procedimientos para la extracción de aceites esenciales en plantas aromáticas. La Paz, Baja California Sur, México: Centro de Investigaciones Biológicas del Noroeste, S.C..

Trávez, V. (2015). Adaptabilidad de hỉbridos Inter-específicos (Oleífera x Guineensis) de palma aceitera en la zona de Santo Domingo quinto año. (Tesis de grado). Universidad Central del Ecuador. Quito, Ecuador.

Vieira, R., Lopes, R., Carvalho, R., Alves, W., Texeira, P., Barcelos, E., Lobato, M. y De Almeida, S. (2012). Domestication and breeding of the American oil palm. En Domestication and breeding: Amazon species. Capítulo 14. Suprema, Viçosa, Brasil. 275-296. 